\title{
Postmortem Study of Organ-Specific Toxicity in Glioblastoma Patients Treated with a Combination of Temozolomide, Irinotecan and Bevacizumab
}

\section{Guangrong Lu}

University of Texas McGovern Medical School: The University of Texas Health Science Center at Houston John P and Katherine G McGovern Medical School https://orcid.org/0000-0002-6887-4789

\section{Mayank Rao}

University of Texas McGovern Medical School: The University of Texas Health Science Center at Houston John P and Katherine G McGovern Medical School

\section{Ping Zhu}

University of Texas McGovern Medical School: The University of Texas Health Science Center at Houston John P and Katherine G McGovern Medical School

\section{Nadine Linendoll}

Tufts Medical Center

\section{Maximilian L. Buja}

University of Texas McGovern Medical School: The University of Texas Health Science Center at Houston John P and Katherine G McGovern Medical School

\section{Meenakshi B. Bhattacharjee}

University of Texas McGovern Medical School: The University of Texas Health Science Center at Houston John P and Katherine G McGovern Medical School

\section{Leomar Y. Ballester}

University of Texas McGovern Medical School: The University of Texas Health Science Center at Houston John P and Katherine G McGovern Medical School

\section{Xuejun Tian}

Tufts Medical Center

\section{Monika Pilichowska}

Tufts Medical Center

\section{Julian K. Wu}

Tufts Medical Center

\section{Georgene W. Hergenroeder}

University of Texas McGovern Medical School: The University of Texas Health Science Center at Houston John P and Katherine G McGovern Medical School 
University of Texas McGovern Medical School: The University of Texas Health Science Center at Houston John P and Katherine G McGovern Medical School

\section{Lei Chen}

University of Texas McGovern Medical School: The University of Texas Health Science Center at Houston John P and Katherine G McGovern Medical School

\section{Rongzhen Zhang}

University of Texas McGovern Medical School: The University of Texas Health Science Center at Houston John P and Katherine G McGovern Medical School

\section{Anil K. Pillai}

University of Texas McGovern Medical School: The University of Texas Health Science Center at Houston John P and Katherine G McGovern Medical School

\section{Robert L. Hunter}

University of Texas McGovern Medical School: The University of Texas Health Science Center at Houston John P and Katherine G McGovern Medical School

\section{Jay-Jiguang Zhu ( $\nabla_{\text {jay.jiguang.zhu@uth.tmc.edu ) }}$}

University of Texas McGovern Medical School: The University of Texas Health Science Center at Houston John P and Katherine G McGovern Medical School https://orcid.org/0000-0003-4635-0914

\section{Research Article}

Keywords: Autopsy, postmortem, end-organ toxicity, chemotherapy, Temozolomide, Irinotecan, Bevacizumab, Glioblastoma, Glioma

Posted Date: January 13th, 2022

DOI: https://doi.org/10.21203/rs.3.rs-1214765/v1

License: (c) (1) This work is licensed under a Creative Commons Attribution 4.0 International License. Read Full License 


\section{Abstract \\ Purpose}

Systemic monotherapy with temozolomide (TMZ) or bevacizumab (BEV); two-drug combinations, such as Irinotecan (IRI) and BEV, TMZ and BEV and a three-drug combination with TMZ, IRI and BEV (TIB) have been used in treating patients with progressive high-grade gliomas including glioblastoma. Most patients tolerated these regimens well with well-established sides effects of hypertension, proteinuria, and reversible clinical myelosuppression (CM). However, organ-specific toxicities have never been examined by postmortem studies.

\section{Methods}

Postmortem tissues (from all major organs) were prospectively collected and examined by standard institution autopsy and brain cutting procedures from 76 decedents, including gliomas $(N=68,44 / \mathrm{M}$, and $24 / F)$ and brain metastases ( $N=8,5 / M$, and 3/F) between 2009 and 2019. Standard hematoxylin and eosin (H\&E) were performed on all major organs and brain samples harvested. Electronic microscopic (EM) study was carried on selected subjects kidney samples per standard EM protocol.

\section{Results}

Twenty-four glioma subjects were treated with TIB [median: 5.5 (range: 1-25) cycles] at glioma recurrence. Exposure to IRI significantly increased the frequency of $\mathrm{CM}(\mathrm{p}=0.05)$. No unexpected adverse events were detected clinically or permenant end-organ damage by postmortem examination among subjects who received TIB compared to subjects who received standard of care (SOC) therapies. Among glioma decedents, the most common causes of death (COD) were tumor progression $(63.2 \%, N=43)$, followed by aspiration pneumonia $(48.5 \%, \mathrm{~N}=33)$. No COD was attributed to acute toxicity from TIB. The study also demonstrated that postmortem kidney specimen is unsuitable for studying renal ultrastructural pathological changes due to autolysis.

\section{Conclusion}

IRI, but not the extended use of TMZ, significantly increased $C M$ in recurrent glioma patients. There is no permanent organ-specific toxicity among glioma decedents who received prolonged BEV, TMZ or TIB regimen based chemotherapies except expected occasional myelosuppresson. COD are most commonly resulted from glioma tumor progression and aspiration pneumonia.

\section{Introduction}


Glioblastoma (GBM) is the most common primary malignant brain tumor with an extremely poor prognosis. The median overall survival (mOS) ranges from $14.6^{1}$ to $23.1^{2}$ months based on phase III clinical trials and population analysis-reported mOS is about 11 months. ${ }^{3}$ The disease usually recurs within 3.3-8.2 months while on active treatments. ${ }^{4}$ There has been no uniform standard of care (SOC) for recurrent GBM (rGBM). For example, BEV (Avastin $\left.{ }^{\circledR}\right)$ is commonly used in the USA but rGBM is not an approved indication for BEV in the European Union. ${ }^{5}$ Unfortunately, no new therapeutic drug has been demonstrated improving mOS of GBM patients in phase III clinical studies in the last decade compared to established therapies. ${ }^{6-8}$

Two-drug combinations, temozolomide (TMZ)/BEV, irinotecan (IRI)/BEV and TMZ/IRI, have been studied in adult rGBM. However, none of these combinations were further evaluated in phase III studies. ${ }^{5,9,10} \mathrm{~A}$ phase III clinical trial using BEV with lomustine (CCNU) $(\mathrm{N}=288)$ for rGBM failed to demonstrate an overall survival advantage over CCNU monotherapy $(\mathrm{N}=149) .{ }^{7}$ In the pediatric oncology community, the TMZ/IRI combination is adopted as an effective "backbone" regimen. ${ }^{11}$ Combination therapy with three drugs $\mathrm{TMZ/BEV/IRI} \mathrm{(TIB)} \mathrm{was} \mathrm{studied} \mathrm{in} \mathrm{clinical} \mathrm{trials} \mathrm{for} \mathrm{treating} \mathrm{pediatric} \mathrm{diseases}{ }^{12-15}$ and gliomas: 1) a phase I study of unresectable GBM $(\mathrm{N}=41) ;{ }^{16}$ 2) a phase II study of newly diagnosed GBM $(\mathrm{N}=75) ;{ }^{17}$ and 3) a phase I study of high-grade glioma $(\mathrm{N}=12) .{ }^{18} \mathrm{~A}$ retrospective study of two institutions' results demonstrated an improved mOS among rGBM patients receiving TIB plus Tumor Treating Fields (TTFields) therapy ( $\mathrm{N}=18)$ compared to those receiving non-TIB BEV-based chemotherapies with TTFields $(\mathrm{N}=30)$ without unexpected side effects. ${ }^{17}$ All these studies concluded that the TIB regimens were welltolerated without unforeseen toxicities. ${ }^{12-19}$ The safety profiles support the TIB regimen administration in rGBM patients with normal bone marrow function since rGBM has limited treatment options.

$B E V$ is a recombinant humanized monoclonal antibody against vascular endothelial growth factor-A. The most common and predictable side effects from its long-term use are hypertension (HTN) and proteinuria. It is postulated that kidney injury from long-term use of BEV is related to the development of HTN and proteinuria, but few renal biopsies in patients who developed BEV-related HTN and proteinuria have been carried out. ${ }^{20}$ The standard duration of TMZ chemotherapy for GBM is six weeks of daily therapy with concurrent fractionated radiation followed by six cycles of adjuvant treatment per the Stupp protocol ${ }^{1}$. It is not uncommon for some GBM patients to take up to 12 cycles of TMZ (prolonged duration) or even longer if there is no evidence of GBM progression or severe side effects. However, there is concern that prolonged use of $\mathrm{TMZ}$ alone or with other chemotherapy drugs may produce more harm than benefit, including alkylating agent-related leukemia. ${ }^{21,22}$

At Tufts Medical Center and Memorial Hermann Hospital (MHH)-Texas Medical Center (TMC)/UTHealth, the TIB regimen was offered to rGBM patients with normal bone marrow function. Treatment decisions were based upon the physician's best knowledge and judgment along with close monitoring of adverse events, balancing the need to control the aggressive tumor and manageable side effects. Clinical side effects from individual chemotherapy drugs, including TMZ, BEV, IRI, and a combination of TMZ/IRI, have been well-documented by the Cancer Therapy Evaluation Program (CTEP). However, there are few reports 
on the side effects from long-term use of TIB, BEV/TMZ, or TMZ monotherapy beyond 12 months. Autopsy studies of $117 \mathrm{GBM}^{23}$ and 40 cases of brain stem gliomas ${ }^{24}$ did not report chemotherapy-related end-organ toxicity. This study aimed to determine any significant end-organ damage from TIB therapy, prolonged use of TMZ monotherapy or TMZ based two-drug therapy. We also wanted to identify pathological changes of the kidney post BEV therapy by standard postmortem examination plus electron microscopic (EM) study of kidney specimens prospectively. From 2009 to 2019,76 deceased subjects were examined in this study, including 24 patients treated with TIB.

\section{Subjects And Methods}

\section{Regulatory approval and consent}

The study protocol and consent received approvals from the Health Sciences Institutional Review Board at Tufts Medical Center and the Committee for the Protection of Human Subjects (CPHS) at Memorial Hermann Hospital (MHH) at Texas Medical Center (TMC) and UTHealth (HSC-MS-11-0133), respectively. Consents for autopsy were obtained from patients antemortem or next of kin postmortem.

\section{Autopsy, brain cutting and EM examinations}

Twelve decedents from Tufts Medical Center and 64 decedents from the MHH-TMC/UTHealth were enrolled in the study between 2009 and 2019 prospectively. Autopsies and brain cutting were done at the decedents' respective institution per the institution's standard of practice (SOP) including unrestricted autopsies $(\mathrm{N}=69)$, restricted autopsies to the brain $(\mathrm{N}=6)$, and to brain and kidney $(\mathrm{N}=1)$. Eight brain metastasis (BM) decedents served as non-glioma references. Most of the decedents' bodies were transported to the morgue within two to four hours. Bodies were stored at $+4^{\circ} \mathrm{C}$ until autopsy. Postmortem organ studies included gross organ examination and microscopic tissue evaluation with hematoxylin and eosin (H\&E) staining per SOP. Kidney tissues from 15 subjects, including ultrasound-guided biopsies in four cadavers before the full autopsy, were analyzed by EM. The tissues destined for EM examination were collected in formaldehyde fixative solution and then prepared per EM examination SOP. Cadaver kidney biopsies were performed with ultrasound guidance by an interventional radiologist (AKP) prior to autopsy to shorten the delay of kidney sample collection. EM slides were reviewed by EM trained nephropathologists (WFG and MP). Three decedents that never received BEV (\#31, \#49, and \#60) were included as controls. The brain was harvested at the time of autopsy and placed in the fixative solution until brain cutting which ranged from 3-6 weeks. Brain cutting was performed per SOP and included gross inspection and microscopic examination with H\&E stain. Fresh samples from each major organ and selected locations of the brain were also collected. The samples were frozen with liquid nitrogen and then stored at $-80^{\circ} \mathrm{C}$ for other use.

\section{Medical record review and side effects grading}

Medical records were reviewed, including operative reports, lab results, MRI image reports, pathology and autopsy reports of body and brain cutting, chemotherapy received and documented adverse events. 
Clinical notes were reviewed to extract age, gender, body weight, diagnosis, overall survival, and adverse events (AEs). Common Terminology Criteria for Adverse Events (CTCAE), version 4.03, was used for grading. Hospitalization and death were considered severe AE (SAE).

\section{Chemotherapy regimens}

Each TIB cycle consists of one 5-day period of TMZ (150 mg/M², PO, QD during day 1-5 every 28 days), two BEV $(10 \mathrm{mg} / \mathrm{kg})$, and two IRI $\left(125 \mathrm{mg} / \mathrm{M}^{2}\right)$ infusions every $14 \pm 2$ days. Each cycle lasts $28-32$ days and allows for the delay of either TMZ or IRI or both based on lab results before administration of chemotherapy per treating physicians. No patient was on p450 enzyme-inducing anti-seizure medication. TIB was offered to rGBM patients for up to 12 cycles, depending on the patient's tolerance. Four patients received more than 12 cycles either continuously or restarted following disease progressions. When TMZ was used for rGBM, but not as a component of the TIB regimen, it was first administered at $200 \mathrm{mg} / \mathrm{m}^{2}$, $\mathrm{PO}, \mathrm{QD}$ on days 1-5 of a 28-day cycle, and then the dose was reduced to $150 \mathrm{mg} / \mathrm{m}^{2}$ if grade III or higher $\mathrm{CM}$ was observed. Detailed chemo/anti-angiogenesis therapy records from each patient are provided in the supplement (Supp Table I).

\section{Grouping based on therapies received}

Decedents with glioma were separated into four groups: Group 1: no chemotherapy beyond concurrent chemoradiation (CCRT), or NCBC $(\mathrm{N}=7)$, including three subjects never received chemotherapy; Group 2: received 1-5 cycles of TIB ( $N=12)$; Group 3: received 6-25 cycles of TIB (N=12); Group 4: SOC group $(\mathrm{N}=37)$. Group 4 included eight patients on maintenance TMZ only; the other 29 received BEV alone or $\mathrm{BEV} / \mathrm{IRI}$ doublet therapies, including three patients who discontinued TMZ during CCRT. Cancer treatment(s) for decedent groups are summarized in Table 2 and detail of drugs for individuals are provided in supplemental Table 1.

\section{Clinical myelosuppression}

Clinical myelosuppression score (CMS), the sum of the highest grade of AEs based on white blood cell count, hemoglobin or platelet lab results, respectively. Grade 1 and 2 AEs do not receive scores, grade III, IV or V AEs are assigned with scores of 3, 4 or 5, repectively. For example, subject 36 (supplement table) experienced grade IV AEs of leukocytopenia and thrombocytopenia but without worse than grade II anemia, so her CMS was $4+4+0=8$. CMS was analyzed based on the number of TMZ cycles: reference (16 cycles) and four subgroups (7-12, 13-18, 19-24, and 25-47 cycles). In addition, CMS was compared in patients who received TIB (cycles: 1-5, 6-25, and 1-25) vs. Group 4, which included various numbers of IRI infusions $(0,1-6,7-52)$.

\section{Statistical analysis}

Univariable binary logistic regression models were performed to explore the associations between different treatment strategies and incident myelosuppression. Odds ratios (OR) and 95\% confidence 
intervals (95\% Cls) are presented. A swimmer plot was utilized to illustrate and compare the timelines of patient history by treatment group. Statistical analyses were performed using Stata IC 15.1 (StataCorp, College Station, TX). $P$ values are two-sided and are considered statistically significant when $P<0.05$.

\section{Results}

\section{Clinical and pathological characteristics of patients}

A total of 76 decedents (49 males, ages 23-80 years old at initial diagnosis, were enrolled prospectively with initial diagnoses as GBM $(\mathrm{N}=53)$, grade III glioma $(\mathrm{N}=8)$, brain metastasis $(\mathrm{BM}, \mathrm{N}=8)$, grade II glioma $(\mathrm{N}=5)$, and spinal glioma ( $\mathrm{N}=2)$ (grades III and IV, respectively). At autopsy, 4 grade II and 5 grade III gliomas had progressed to secondary GBMs (Table 1). The swimmer plot is presented in Figure 1. The average time from death to postmortem examination at MHH-TMC/UTHealth was $35.3 \pm 3.7$ hours (range: 7-100 hours, calculated from 38 cases with accurate documentation of timing of death).

\section{Kidney ultrastructure examination}

EM studies were performed in 14 subjects to understand the ultrastructural changes in those who had HTN and proteinuria due to BEV therapy. An interval from death to tissue collection was not available from five decedents at Tufts Medical Center; the interval from death to tissue collection was $19.8 \pm 7.5$ hours from nine glioma decedents at $\mathrm{MHH}-\mathrm{TMC} / \mathrm{UTHealth}$. To differentiate true microscopic findings from possible artifacts from autolysis, interventional radiologist (AKP) biopsied cadaver kidney under ultrasound guidance on 4 decedents \#22 (3 hrs), \#20 (6 hrs), \#17 (7 hrs), and \#49 (9 hrs). The EM results of podocyte effacement with basement membrane detachment were observed in subjects exposed to $\mathrm{BEV}$ and naïve to BEV. making observation inconclusive due to autolysis.

\section{Clinical myelosuppression on active therapies}

Analysis showed that $41.7 \%(5 / 12), 16.7 \%(2 / 12)$, and $20 \%$ (10/37) of decedents experienced grade III or worse $\mathrm{CM}$ during active therapies in groups 2,3 , and 4, respectively. Compared to group 4, ORs were 1.93 (95\% Cl: $0.50-7.50, P=0.343$ ) and 0.54 (95\% Cl: $0.10-2.91, P=0.473$ ) for groups 2 and 3 , respectively (Table $3)$. The $\mathrm{CM}$ rates were $57.1 \%$ and $47.6 \%$ in IRI infusion subgroups who received $1-6$ cycles $(\mathrm{N}=14)$ and 112 cycles $(\mathrm{N}=21)$, respectively. The ORs in these two groups were $7.67(95 \% \mathrm{Cl}: 1.71-34.33, \mathrm{P}=0.008)$ and 5.23 (95\% Cl: 1.34-20.45, P=0.018) (Table 3). By contrast, the likelihoods of CM for patients treated with 7-12, 13-18, 19-24, and 25-47 cycles of TMZ were not significantly increased compared to those receiving 1-6 cycles (all P>0.05) (Table 3 ).

\section{Bone marrow examinations at autopsy}

Bone marrow exams were performed in 39 decedents. BM study in 4 decedents (case \#9, 46, 65 and 69) showed as hypocellular, but not conclusive if they are appropriate for age or indicative of treatmentrelated changes. Nine severe hypocellularity cases were identified: two from group 2 (case \#17 and 23), three from group 4 (case \#29, 52 and 59), and four from the BM group (case \#71, 73, 74 and 76). 
Three severe hypocellular bone marrow specimens (\#17, 23, and 52) matched antemortem CM history. Subject \# 36's (group 4, SOC) bone marrow, who had a history of leukocytopenia and thrombocytopenia, was hypercellular. There was no evidence of postmortem BM abnormality among decedents in groups 1 and 3 .

Five patients (\#29, 32, 38, 62, and 63) discontinued TMZ due to severe CM during CCRT. But CM in glioma patients did not develop permanent bone marrow damage as often as seen among BM decedents treated with chemotherapy. Patients exposed to extended TMZ (Figure 2C) or TIB regimens (Figure 2D) still had normal bone marrow.

\section{Other clinical adverse events}

The three most common AEs in group 4 were grade III HTN (37.5\%), weight loss (29.7\%), and venous thromboembolism (VTE, 24.3\%). Grade III HTN (41.7\%) was also the most common AE among patients who received TIB (groups 2 and 3 combined). The grade III CM was $33.3 \%$ and $21.6 \%$ among TIB and group 4, respectively. However, no grade IV thrombocytopenia was observed in the TIB group, while the rate was $13.5 \%$ in group 4 . All AEs grade III or above are listed in Table 2.

\section{Cause of death (COD) for recurrent/progressive glioma patients including GBM patients}

Among the 68 glioma decedents, disease progression was the most common COD ( $N=43,63.2 \%)$ (Table 2). The second most common COD was aspiration pneumonia ( $\mathrm{N}=33,48.5 \%)$ among glioma cases. Herniation with brain swelling was detected in 12 (17.6\%) subjects, while cerebral or intraventricular hemorrhages were documented in 7 glioma cases. Uncommon CODs include decedent \#67, who died at home from sudden death with autopsy finding of cardiomyopathy; descendants \#26 and \#76 expired from cerebral herniation soon after decompression surgeries. Three subjects (\#52, 57, and 60) passed away in hospital due to severe adverse events while on active anti-cancer treatments.

\section{Discussion}

Postmortem examination is the gold standard to identify end-organ toxicities from chemotherapies. ${ }^{25}$ In this study, we documented clinical AEs and autopsy findings from 76 decedents, including 68 glioma patients with the majority of diagnosis as de novo GBM, to study the possible sequelae of TIB chemotherapy-related toxicity. Hypertension (41.7\%) and combined CM (33.3\%) were the two most common AEs among patients who received TIB treatment. In contrast, hypertension (32.1\%), weight loss (29.7\%), and VTE (24.3\%) were the most common AEs among patients treated with SOC. We found that IRI, but not the extended TMZ cycles, significantly increased the likelihood of CM among rGBM patients. Long-term use of TIB (up to 25 cycles) did not cause unexpected toxicities or end-organ injuries among rGBM patients. To limit toxic drug effects from IRI, we recommend screening patients for the polymorphism of the UDP-glucuronosyl-transferase 1A1 (UGT1A1) locus ${ }^{17}$ and closely monitoring bone marrow function when an IRI-containing regimen is considered. 
TMZ-induced myelosuppression is a known side effect and was reported predominantly in female patients. ${ }^{26}$ Five $(7.7 \%)$ decedents had to discontinue TMZ permanently after brief exposure to low daily doses during the CCRT period because of severe myelosuppression. Most oncologists discontinue TMZ for rGBM based on the assumption that TMZ will no longer be effective. However, $101^{27}$ and $108^{28}$ cycles of TMZ had been used to treat recurrent glioma patients without reports of severe myelosuppression. In the current study, up to 47 cycles of TMZ and 25 cycles of TIB were not associated with an increased risk of developing $\mathrm{CM}$ among recurrent glioma patients.

Bone marrow from $3(\# 17,23$, and 52) glioma decedents demonstrated chemotherapy-related hypocellularity with documented clinical histories of CM. An analysis revealed that infusion with 1-6 $(\mathrm{N}=14)$ and 1-12 $(\mathrm{N}=21)$ IRI cycles increased the risk of $\mathrm{CM}$ by $7.67(\mathrm{P}=0.008)$ and $5.23(\mathrm{P}=0.018)$ times. CTEP-issued side effects from the TMZ/IRI combination include a 4-20\% chance of developing into irreversible bone marrow damage with the observation that most of the CM events occurred soon after a new treatment drug was started. Genetic variants of the UGT1A1 gene and NADPH dehydrogenase quinone 1 might predispose susceptible patients to develop CM from IRI. ${ }^{29,30,31}$ These genetic factors may explain why some patients developed CM soon after IRI was given, while other patients rarely experienced CM, even with prolonged usage. It is also likely that previous TMZ treatment as adjuvant therapy predisposes patients with an elevated risk for CM when IRI is added into salvage treatment. To reduce overlapping side effects on myelosuppression, TMZ dosage in the TIB regimen was set at 150 $\mathrm{mg} / \mathrm{M}^{2}$ and subjected to further reduction if a given patient could not tolerate the dosage.

According to forensic studies, fast morphological changes (autolysis, degenerative) at the microscopic and ultrastructural level have been reported in human ${ }^{32}$ and animal ${ }^{33}$ kidneys within hours of death. Variable degrees of autolysis in kidney specimens (supplement Figure S1) prevented us from studying glomerular ultrastructural changes at autopsy. Meanwhile, ultrastructural changes such as unique hyaline occlusive glomerular microangiopathy were demonstrated through renal biopsy from patients actively treated with BEV. ${ }^{34}$ There was no evidence of fibrin polymers or extensive loss of podocyte foot processes, ${ }^{34}$ which was quite a difference from acute or chronic thrombotic microangiopathy cases.

The current study concluded that rGBM patients predominantly died directly from respiratory dysfunction, secondary to incapacitation from tumor progression (supplement Table 2). Death from brain herniation is not as common today as was reported in the 1990 's ${ }^{26}$ due to treatment advances, such as repeated debulking surgery, ventriculoperitoneal shunt placement, BEV, and steroid use.

This study has its limitations: 1) Toxicity to male and female reproductive organs was not evaluated; 2) The cohort was not selected by pre-specified criteria. Medical records review and AE/SAE assessment are retrospective analyses which may have introduced selection bias; 3 ) There were variable intervals from death to tissue collection 4) Personalized and targeted treatments in addition to TMZ, BEV, and/or IRI are potential confounding factors; 5) There may be patient selection bias since subjects received the TIB regimen dictated by normal or near-normal bone marrow function before TIB therapy. 
In summary, the TIB regimen might have caused a higher rate of manageable CM than those seen from patients on SOC therapy. This difference is likely due to IRI involvement rather than the extended use of TMZ or BEV. There were no unexpected clinical adverse effects or organ-specific toxicities by postmortem examinations, even with the long-term use of TIB. We demonstrated that GBM disease progression and aspiration pneumonia are the most common CODs among glioma decedents. This result will allow physicians to plan patients' treatment regimens and design clinical trials with BEV and BEV-based therapies without the fear of the high risk of end-organ damages, besides side effects clinically known.

\section{Declarations}

Funding source: This study is partially funded by the Dr. Marnie Rose Foundation to J-J.Z.

Disclosure of potential conflicts of interest: The authors have no relevant financial or non-financial interests to disclose.

Research involving human participants: This study used postmortem human specimens and it was performed in line with the principles of the Declaration of Helsinki. It is approved by the Health Sciences Institutional Review Board (IRB) at Tufts Medical Center. The same study protocol was also approved by the Committee for the Protection of Human Subjects (CPHS) at the University of Texas Health Science Center at Houston (UTHealth) (HSC-MS-11-0133).

Informed consent: Consents for autopsy examination were obtained from all participants, either from patients when patients were alive or from the next of kin after subjects had deceased [full autopsies $(\mathrm{N}=69)$, restricted autopsies to the brain $(\mathrm{N}=6)$ and restricted to the brain and kidney $(\mathrm{N}=1)]$.

Author Contributions: All authors contributed to the study. J-J. Zhu initiated the project and mobilized resources for the study. JK. Wu and J-J Zhu were investigators at two institutes. Autopsies and tissue collections were performeded or supervised by M. Buja, MB. Bhattacharjee, LY. Ballester, X. Tian, M. Pilichowska, GW. Hergenroeder, R. Zhang, and RL. Hunter. Ultrasound guided kidney biopsy in cadaver was performed by AK. Pillai. Slide review and pathology reports were issued by M. Buja, MB. Bhattacharjee, LY. Ballester, X. Tian, M. Pilichowska, RL. Hunter. WF. Glass and L. Chen. Clinial data collection and analysis were preformed by G. Lu, M. Rao, P. Zhu, N. Linendoll and J-J. Zhu. The first draft of the manuscript was written by G. Lu and all authors commented on previous versions of the manuscript. All authors read and approved the final manuscript.

Acknowledgment: We are very grateful to the pathology attending physicians and residents from both Tufts Medical Center and UTHealth McGovern Medical School/Memorial Hermann Hospital at Texas Medical Center, respectively. We appreciate the technical assistance of sample preparation for electron microscope examination by Ms. Patricia Navarro at the Pathology department of UTHealth. We are very thankful to Drs. Bihong Zhao and Joanna S. O'Leary at UTHealth for their editorial assistance with this manuscript. 
This manuscript has processed data included as electronic supplementary material. The datasets (raw data) generated during and/or analysed during the current study are available from the corresponding author on reasonable request.

\section{References}

1. Stupp R, Mason WP, van den Bent MJ et al (2005) : Radiotherapy plus concomitant and adjuvant temozolomide for glioblastoma. N Engl J Med 352:987-996,

2. Liau LM, Ashkan K, Tran DD et al (2018) : First results on survival from a large Phase 3 clinical trial of an autologous dendritic cell vaccine in newly diagnosed glioblastoma. J Transl Med 16:142,

3. Zhu P, Du XL, Lu G et al (2017) : Survival benefit of glioblastoma patients after FDA approval of temozolomide concomitant with radiation and bevacizumab: A population-based study. Oncotarget 8:44015-44031,

4. Stupp R, Taillibert S, Kanner A et al (2017) : Effect of Tumor-Treating Fields Plus Maintenance Temozolomide vs Maintenance Temozolomide Alone on Survival in Patients With Glioblastoma: A Randomized Clinical Trial. JAMA 318:2306-2316,

5. Seystahl K, Wick W, Weller M (2016) : Therapeutic options in recurrent glioblastoma-An update. Crit Rev Oncol Hematol 99:389-408,

6. Stupp R, Wong ET, Kanner AA et al (2012) : NovoTTF-100A versus physician's choice chemotherapy in recurrent glioblastoma: a randomised phase III trial of a novel treatment modality. Eur $\mathrm{J}$ Cancer 48:2192-2202,

7. Wick W, Gorlia T, Bendszus M et al (2017) : Lomustine and Bevacizumab in Progressive Glioblastoma. N Engl J Med 377:1954-1963,

8. Roth P, Gorlia T, Reijneveld JC et al (2021) : EORTC 1709/CCTG CE.8: A phase III trial of marizomib in combination with temozolomide-based radiochemotherapy versus temozolomide-based radiochemotherapy alone in patients with newly diagnosed glioblastoma. JCO 39:2004-2004,

9. Weller M, Cloughesy T, Perry JR et al (2013) : Standards of care for treatment of recurrent glioblastoma-are we there yet? Neuro Oncol 15:4-27,

10. Gilbert MR, Pugh SL, Aldape K et al (2017) : NRG oncology RTOG 0625: a randomized phase II trial of bevacizumab with either irinotecan or dose-dense temozolomide in recurrent glioblastoma. $J$ Neurooncol 131:193-199,

11. Bagatell R, London WB, Wagner LM et al (2011) : Phase II study of irinotecan and temozolomide in children with relapsed or refractory neuroblastoma: a Children's Oncology Group study. J Clin Oncol 29:208-213,

12. Levy A, Krailo M, Chi S et al (2017) : PDCT-09. Temozolomide with irinotecan versus temozolomide, irinotecan plus bevacizumab for recurrent medulloblastoma/cns pnet of childhood: report of a cog randomized phase ii screening trial. Neuro Oncol 19:vi186, 
13. Magnan HD, Price A, Chou AJ et al (2017) : A pilot trial of irinotecan, temozolomide and bevacizumab (ITB) for treatment of newly diagnosed patients with desmoplastic small round cell tumor (DSRCT). JCO 35:11050-11050,

14. Venkatramani R, Malogolowkin M, Davidson TB et al (2013) : A phase I study of vincristine, irinotecan, temozolomide and bevacizumab (vitb) in pediatric patients with relapsed solid tumors. PLoS One 8:e68416,

15. Modak S, Kushner BH, Basu E et al (2017) : Combination of bevacizumab, irinotecan, and temozolomide for refractory or relapsed neuroblastoma: Results of a phase II study. Pediatr Blood Cancer 64,

16. Peters KB, Lou E, Desjardins A et al (2015) : Phase II Trial of Upfront Bevacizumab, Irinotecan, and Temozolomide for Unresectable Glioblastoma. Oncologist 20:727-728,

17. Vredenburgh JJ, Desjardins A, Reardon DA et al (2011) : The addition of bevacizumab to standard radiation therapy and temozolomide followed by bevacizumab, temozolomide, and irinotecan for newly diagnosed glioblastoma. Clin Cancer Res 17:4119-4124,

18. Hummel TR, Salloum R, Drissi R et al (2016) : A pilot study of bevacizumab-based therapy in patients with newly diagnosed high-grade gliomas and diffuse intrinsic pontine gliomas. J Neurooncol 127:53-61,

19. Aguilera D, Mazewski C, Fangusaro J et al (2013) : Response to bevacizumab, irinotecan, and temozolomide in children with relapsed medulloblastoma: a multi-institutional experience. Childs Nerv Syst 29:589-596,

20. Shye M, Hanna RM, Patel SS et al (2020) : Worsening proteinuria and renal function after intravitreal vascular endothelial growth factor blockade for diabetic proliferative retinopathy. Clin Kidney $\mathrm{J}$ 13:969-980,

21. Perry JR, Brown MT, Gockerman JP (1998) : Acute leukemia following treatment of malignant glioma. J Neurooncol 40:39-46,

22. Liu P, Li P, Lei T et al (2018) : Acute lymphoblastic leukemia following temozolomide treatment in a patient with glioblastoma: A case report and review of the literature. Oncol Lett 15:8663-8668,

23. Silbergeld DL, Rostomily RC, Alvord EC (1991) : The cause of death in patients with glioblastoma is multifactorial: clinical factors and autopsy findings in 117 cases of supratentorial glioblastoma in adults. J Neurooncol 10:179-185,

24. Yoshimura J, Onda K, Tanaka R et al Clinicopathological study of diffuse type brainstem gliomas: analysis of 40 autopsy cases. Neurol Med Chir (Tokyo) 43:375-382; discussion 382, 2003

25. Penninckx B, Van de Voorde WM, Casado A et al (2012) : A systemic review of toxic death in clinical oncology trials: an Achilles' heel in safety reporting revisited. $\mathrm{Br} \mathrm{J}$ Cancer 107:1-6,

26. Kourelis TV, Buckner JC, Gangat $\mathrm{N}$ et al (2015) : Temozolomide induced bone marrow Suppression--A single institution outcome analysis and review of the literature. Am J Hematol 90:E183-184,

27. Barbagallo GMV, Paratore S, Caltabiano R et al (2014) : Long-term therapy with temozolomide is a feasible option for newly diagnosed glioblastoma: a single-institution experience with as many as 
101 temozolomide cycles. Neurosurg Focus 37:E4,

28. Hirano H, Kawahara T, Niiro M et al (2017) : Anaplastic astrocytoma cells not detectable on autopsy following long-term temozolomide treatment: A case report. Mol Clin Oncol 6:321-326,

29. Tsunedomi R, Hazama S, Fujita Y et al (2014) : A novel system for predicting the toxicity of irinotecan based on statistical pattern recognition with UGT1A genotypes. Int J Oncol 45:1381-1390,

30. Innocenti F, Undevia SD, lyer L et al (2004) : Genetic variants in the UDP-glucuronosyltransferase 1A1 gene predict the risk of severe neutropenia of irinotecan. J Clin Oncol 22:1382-1388,

31. Liu D, Li J, Gao J et al (2017) : Examination of multiple UGT1A and DPYD polymorphisms has limited ability to predict the toxicity and efficacy of metastatic colorectal cancer treated with irinotecan-based chemotherapy: a retrospective analysis. BMC Cancer 17:437,

32. Kushwaha V, Yadav M, Srivastava A et al (2010) : Time since death from degenerative changes in the Kidney. J Indian Acad Forensic Med 32,

33. Tomita Y, Nihira M, Ohno Y et al (2004) : Ultrastructural changes during in situ early postmortem autolysis in kidney, pancreas, liver, heart and skeletal muscle of rats. Legal Medicine 6:25-31,

34. Person F, Rinschen MM, Brix SR et al (2019) : Bevacizumab-associated glomerular microangiopathy. Mod Pathol 32:684-700,

\section{Tables}


Table 1

Summary of patient characteristics

\begin{tabular}{|c|c|c|c|c|c|}
\hline Characteristics & BM & DMG & $\begin{array}{l}\text { Grade II } \\
\text { Glioma }\end{array}$ & $\begin{array}{l}\text { Grade III } \\
\text { Glioma }\end{array}$ & GBM \\
\hline Number of patients at autopsy & 8 & $3^{*}$ & 1 & 2 & $62^{\star \star}$ \\
\hline $\begin{array}{l}\text { Number of patients at initial } \\
\text { diagnosis }\end{array}$ & 8 & $\begin{array}{l}2 \\
\text { (spine) }\end{array}$ & 5 & 8 & 53 \\
\hline $\begin{array}{l}\text { Age at diagnosis (median, } \\
\text { range) }\end{array}$ & $\begin{array}{l}60(39- \\
75)\end{array}$ & $\begin{array}{l}28(23- \\
33)\end{array}$ & $48(31-56)$ & $38.5(27-76)$ & $\begin{array}{l}61(25- \\
80)\end{array}$ \\
\hline Gender (Male/Female) & $5 / 3$ & $0 / 2$ & $4 / 1$ & $5 / 3$ & $35 / 18$ \\
\hline \multicolumn{6}{|l|}{ Race } \\
\hline Caucasian & 7 & 1 & 3 & 8 & 44 \\
\hline African American & 0 & 0 & 0 & 0 & 4 \\
\hline Latino & 1 & 0 & 1 & 0 & 0 \\
\hline Asian & 0 & 1 & 0 & 0 & 2 \\
\hline Unspecified & 0 & 0 & 1 & 0 & 3 \\
\hline
\end{tabular}


Table 2

Occurrence of adverse events (grade III and above)

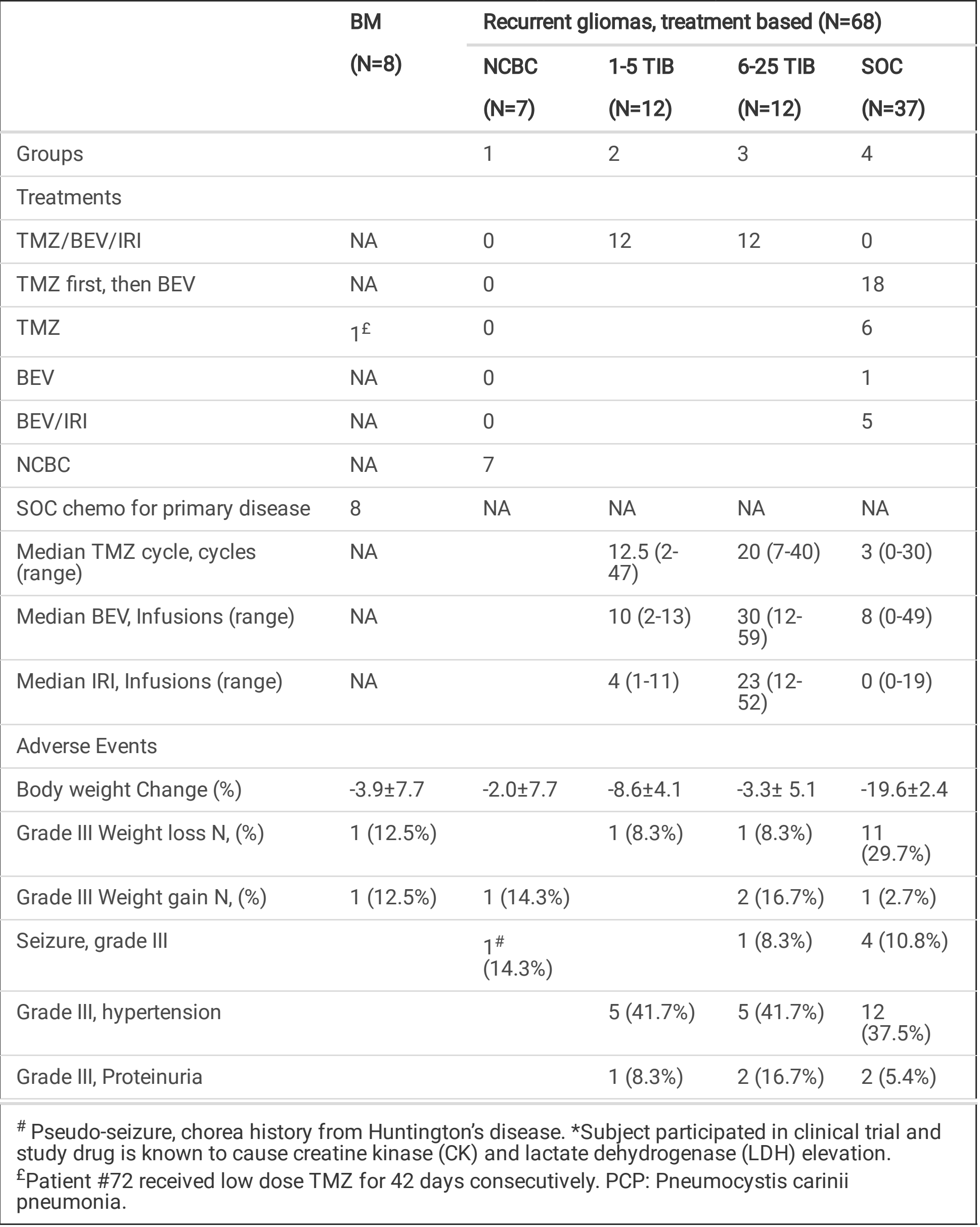




\begin{tabular}{|c|c|c|c|c|c|}
\hline & BM & Recurrent & omas, treat & ent based $(\AA$ & 68) \\
\hline & $(\mathrm{N}=8)$ & NCBC & $1-5 \mathrm{TIB}$ & 6-25 TIB & soc \\
\hline & & $(\mathrm{N}=7)$ & $(\mathrm{N}=12)$ & $(\mathrm{N}=12)$ & $(\mathrm{N}=37)$ \\
\hline Grade III, Anemia & $1(12.5 \%)$ & $1(14.3 \%)$ & $1(8.3 \%)$ & & $4(10.8 \%)$ \\
\hline Grade III, thrombocytopenia & & $1(14.3 \%)$ & $2(16.7 \%)$ & $2(16.7 \%)$ & $2(5.4 \%)$ \\
\hline Grade III, neutropenia & & & $3(25 \%)$ & & $2(5.4 \%)$ \\
\hline Grade IV, hypertension & & & & & $1(2.7 \%)$ \\
\hline Grade IV, thrombocytopenia & $1(12.5 \%)$ & $1(14.3 \%)$ & & & $5(13.5 \%)$ \\
\hline Grade IV, neutropenia & $1(12.5 \%)$ & & & & $3(8.1 \%)$ \\
\hline Grade III, VTE & $1(12.5 \%)$ & & $2(16.7 \%)$ & $1(8.3 \%)$ & $9(24.3 \%)$ \\
\hline Grade V, Perforated diverticulitis & & & & & $1(2.7 \%)$ \\
\hline Grade III, Kidney failure & & $1(14.3 \%)$ & & & \\
\hline Grade IV, Kidney failure & & $1(14.3 \%)$ & & & $1(2.7 \%)$ \\
\hline $\begin{array}{l}\text { Abnormal liver enzymes, grade } \\
\text { IV }\end{array}$ & & & $1(8.3 \%)$ & & $1(2.7 \%)$ \\
\hline CK and LDH elevation, grade III & & & & & $1 *(2.7 \%)$ \\
\hline Abnormal electrolytes, grade III. & & $1(14.3 \%)$ & & $1(8.3 \%)$ & $2(5.4 \%)$ \\
\hline Meningitis, grade III & & & & $1(8.3 \%)$ & \\
\hline PCP, grade IV & & & & & $1(2.7 \%)$ \\
\hline CMV pneumonitis, grade III & $\begin{array}{l}1^{\Omega} \\
(12.5 \%)\end{array}$ & & & & \\
\hline Shingles, grade III & & $1(14.3 \%)$ & & & $1(2.7 \%)$ \\
\hline Cellulitis, grade III & & $1(14.3 \%)$ & & & $1(2.7 \%)$ \\
\hline $\begin{array}{l}\text { \# Pseudo-seizure, chorea history } \\
\text { study drug is known to cause cre } \\
\text { £Patient \#72 received low dose } T \\
\text { pneumonia. }\end{array}$ & $\begin{array}{l}\text { m Huntingt } \\
\text { ne kinase ( } \\
Z \text { for } 42 \text { day }\end{array}$ & $\begin{array}{l}\text { s disease. } \\
\text { and lactat } \\
\text { consecutive }\end{array}$ & $\begin{array}{l}\text { bject parti } \\
\text { lehydroger } \\
\text { PCP: Pneı }\end{array}$ & $\begin{array}{l}\text { ated in clir } \\
\text { e (LDH) el } \\
\text { pcystis car }\end{array}$ & $\begin{array}{l}\text { I trial and } \\
\text { tion. }\end{array}$ \\
\hline
\end{tabular}


Table 3

Risk of myelosuppression rate in relation to varied chemotherapy modalities*.

Myelosuppression rate

Predictors

Total Myelo-

OR $\quad 95 \% \mathrm{Cl}$

$P$

suppression

Standard care vs. $\mathrm{TIB}, \mathrm{N}=61$

Standard care

37

10

1.00

TIB

24

7

1.11

$0.36-3.48$

0.856

Standard care vs. TIB (cutoff: 6 ), $\mathrm{N}=61$

Standard care

37

10

1.00

TIB: $<6$

12

5

1.93

$0.50-7.50$

0.343

TIB: $>=6$

12

2

0.54

$0.10-2.91$

0.473

TMZ cycles: continuous, $\mathbf{N}=58$

TMZ cycles

58

15

0.97

$0.91-1.03$

0.312

TMZ cycles: cutoff $-6,12,18,24 ; N=58$

$1-6$

7-12

13-18

$19-24$

$25-47$

TMZ cycles: cutoff, $12 ; \mathrm{N}=58$

$<12$

$>=12$

TIB cycles: cutoff, $6 ; \mathrm{N}=24$

$<6$

$>=6$

IRI cycles: cutoff, $0 ; \mathrm{N}=61$

0

$>0$

28

10

8

4

8

35

23

12

12

2

27

34

13

$1.00-$

$1.67 \quad 0.37-7.53$

0.507

$\begin{array}{lll}0.36 & 0.04-3.39 & 0.370\end{array}$

$\begin{array}{lll}0.83 & 0.08-9.25 & 0.882\end{array}$

$0.36 \quad 0.04-3.39$

0.370 


\section{Myelosuppression rate}

IRI cycles: cutoff, 0,$6 ; \mathrm{N}=61$

$\begin{array}{llllll}0 & 27 & 4 & 1.00 & - & - \\ <=6 & 14 & 8 & 7.67 & 1.71-34.33 & 0.008 \\ >6 & 20 & 5 & 1.92 & 0.44-8.31 & 0.385\end{array}$

IRI cycles: cutoff, $0,12: \mathrm{N}=61$

$\begin{array}{llllll}0 & 27 & 4 & 1.00 & - & - \\ <=12 & 21 & 10 & 5.23 & 1.34-20.45 & 0.018 \\ >12 & 13 & 3 & 1.72 & 0.32-9.17 & 0.523\end{array}$

Abbreviation: OR, odds ratio; $95 \% \mathrm{Cl}, 95 \%$ confidence interval; TIB, Temozolomide+ Irinotecan+Bevacizumab; TMZ, Temozolomide; IRI, Irinotecan.

* Univariable binary logistic regression models.

Figures 


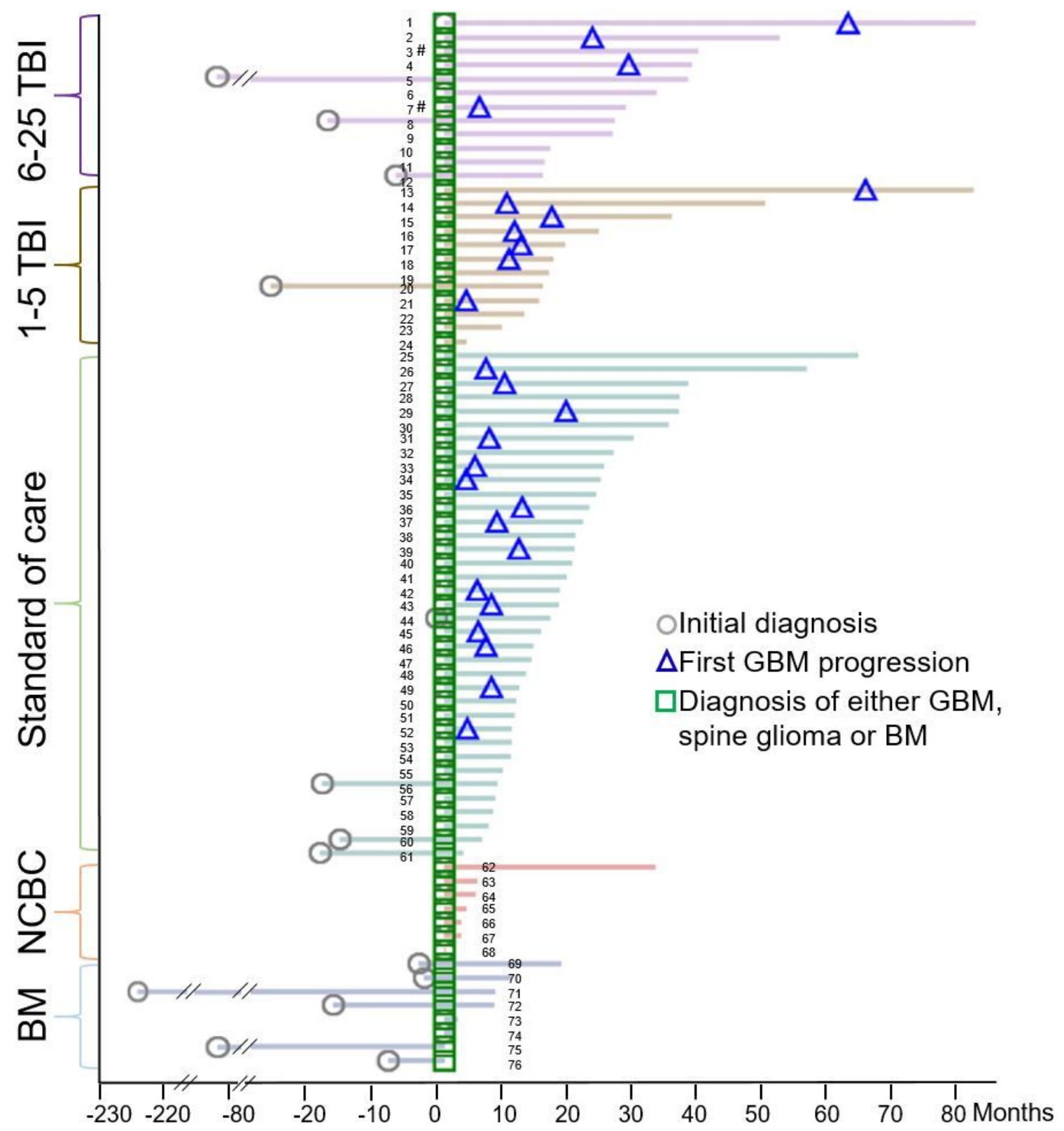

\section{Figure 1}

A swimmer plot showing time points of diagnosis, surgery and overall survival.

All cases were aligned based on a time point when GBM, spinal glioma or BM was first diagnosed, with a few exceptions (\#) being made because their diagnosis was never upgraded to GBM when patients were alive. Time point when lower grade glioma and systemic cancer before the diagnosis of BM were captured to show overall survival time. BM: brain metastasis; NCBC: no chemotherapy beyond CCRT. 
A

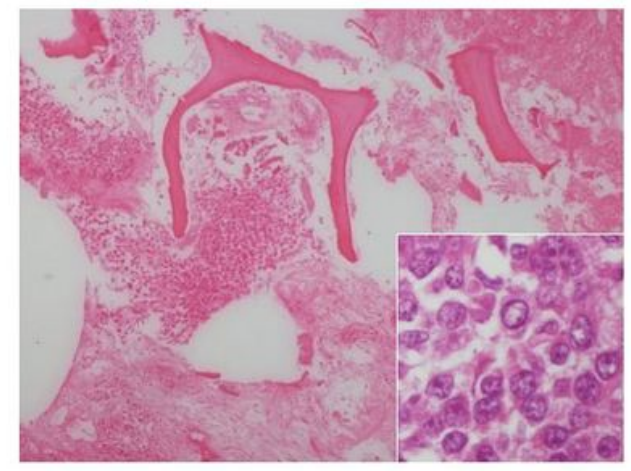

$\mathrm{C}$

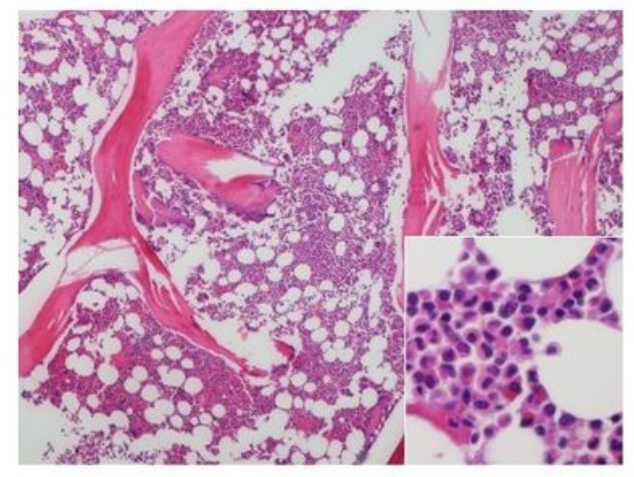

B

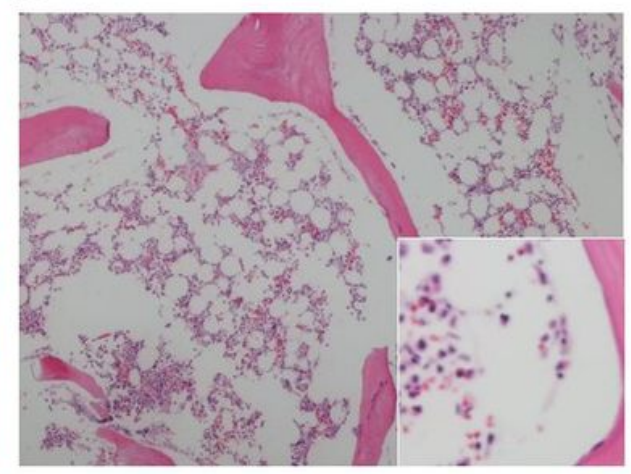

$\mathrm{D}$

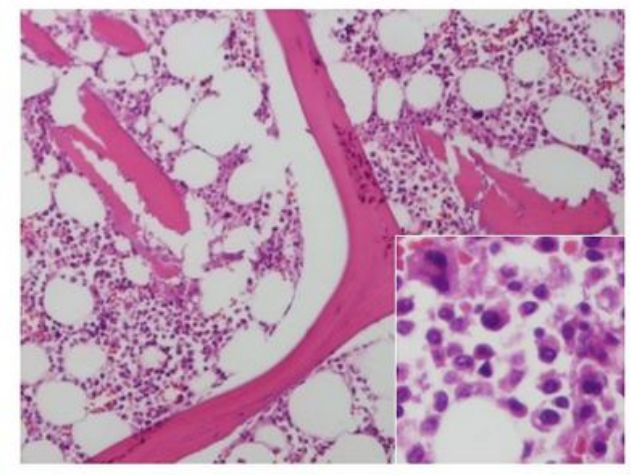

Figure 2

\section{Figure 2}

Bone marrow changes in different diseases and treatment groups. A) Patient \#72 in BM group passed away from a stage IV parotid gland carcinosarcoma. Tumor infiltrate (insert) completely destroyed normal bone marrow cells. B) Patient \#65 (group 1: NCBC) did not receive chemotherapy. Examination of her bone marrow revealed as hypocellular (30-50\%) with trilineage hematopoiesis. C). Patient \#61 (group 4: SOC) was diagnosed of grade III glioma, he was treated with 9 cycles TMZ before disease progression to GBM. He received experimental Toca 511 virus injection into resection cavity wall and took 1 cycle of Toca FC before giving up further treatments. His bone marrow showed as normocellular, $40 \%$ with trilineage hematopoiesis. D) Patient \#9 (group 3: 6-25 TIB) was treated with 7 TIB cycles, 35X BEV and $24 X$ IRI infusions. Her bone marrow contained $40-60 \%$ cellularity with trilineage hematopoiesis. Magnification is $40 \mathrm{X}$; insert is magnified by $200 \mathrm{X}$.

\section{Supplementary Files}

This is a list of supplementary files associated with this preprint. Click to download.

- LuetalSupplementJAN2022.docx 